\title{
SSPE-Risiko ist viel höher als angenommen
}

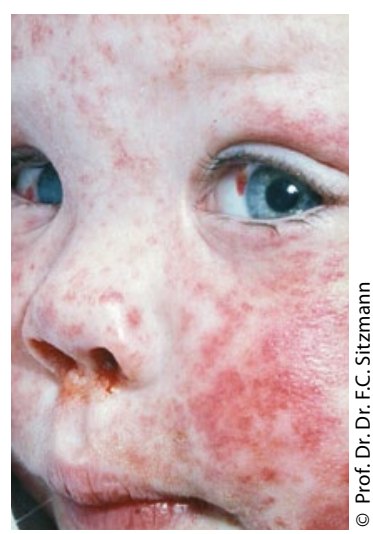

Auch nach überstandener Maserninfektion drohen Komplikationen.
— Die subakute sklerosierende Panenzephalitis (SSPE) ist eine gefürchtete, stets tödlich verlaufende Folgeerkrankung der Maserninfektion. Laut den Zahlen, die Wissenschaftler vom Bayerischen Landesamt für Gesundheit und Lebensmittelsicherheit vorgelegt haben, sind allein zwischen 2003 und 2009 hierzulande 39 Patienten vor ihrem 16. Geburtstag an SSPE erkrankt. Das SSPE-Risiko von Personen, die während ihrer ersten fünf Lebensjahre eine Maserninfektion durchgemacht haben, liegt demnach bei $1: 1700$ bis $1: 3300$. Die Gefahr ist somit weitaus größer als frühere Schätzungen vermuten ließen. In der älteren Literatur ist das Risiko für SSPE mit $1: 100000$ beziffert worden.

Die Forscher bedienten sich für ihre Berechnungen zweier großer Register für SSPE: zum einen der Datenbank der Erhebungseinheit für seltene pädiatrische Erkrankungen in Deutschland (ESPED) an der Universität Düsseldorf, zum anderen der Fallsammlung des Virologischen Instituts der Universität Würzburg. Die Zahl der von 1994-2001 in Deutschland an Masern Erkrankten war mit 43000 zu veranschlagen. Die mediane Latenzzeit zwischen der Maserninfektion und dem Auftreten einer SSPE betrug laut der Erhebung acht Jahre.

\section{So früh wie möglich gegen Masern impfen!}

Die Altersverteilung der Studienpatienten legt nahe, dass das SSPERisiko umso höher ist, je jünger die Kinder zum Zeitpunkt ihrer Maserninfektion sind. Besonders hoch ist die Gefahr in den ersten zwei bis drei Lebensjahren. „Das unterstreicht, wie wichtig es ist, im frühestmöglichen Alter gegen Masern zu impfen", so die Autoren. Derzeit wird die erste Impfdosis gegen Ende des ersten Lebensjahres verabreicht. Jüngere Kinder können nur über die Herdenimmunität vor Masern geschützt werden. Das setzt allerdings eine ausreichende Durchimpfung der Bevölkerung voraus.

In Deutschland ist das nicht erreicht. Aktuelle Daten des Robert Koch-Instituts (RKI) für 2013 weisen bis zum Stichtag 10. Juli 1043 Masernfälle in Deutschland aus. Das sind fast neunmal mehr als 2012. An der Spitze der Masernstatistik stehen Bayern, wo in diesem Jahr bisher 465 Menschen an Masern erkrankt sind, und Berlin mit 387 Fällen. Laut RKI handelt es sich bei rund der Hälfte der Erkrankten um über 20-Jährige. 36\% der Masernpatienten mussten stationär behandelt werden.

$\mathrm{rb}$.

- Schönberger Ket al. Epidemiology of Subacute Sclerosing Panencephalitis (SSPE) in Germany from 2003 to 2009: A Risk Estimation. PLoS ONE 8(7): e68909; doi:10.1371/journal.pone.0068909

\section{Hier steht eine Anzeige.}

\author{
望 Springer
}

